\title{
Mastoplastia de aumento via axilar: avaliação da técnica subfascial
}

\author{
Mammaplasty of increase axillary way: analysis of the subfascial technique
}

\author{
Paulo Rogério \\ QuieregatTo Do Espírito \\ SANTO $^{1}$
}

Trabalho realizado na Clínica privada do autor, São Paulo, SP,

Brasil. Trabalho apresentado

no $43^{\circ}$ Congresso Brasileiro de

Cirurgia Plástica, em Recife, PE,

2006, para ascensão a Membro Titular da Sociedade Brasileira de Cirurgia Plástica.

Artigo submetido pelo SGP (Sistema de Gestão de Publicações) da RBCP.

Artigo recebido: 23/7/2010 Artigo aceito: $22 / 11 / 2010$

\begin{abstract}
RESUMO
Introdução: A cirurgia para aumento de mamas tem evoluído muito nos últimos anos, assim como o grau de exigência de nossas pacientes em relação aos resultados relacionados à forma das mamas e às cicatrizes cada vez menos aparentes. Método: Nossa experiência teve início em novembro de 2002 e demonstra a possibilidade de utilização da técnica via axilar num plano subfascial para aumento de mamas por meio de implantes mamários de silicone gel. Resultados: A técnica empregada é passível de ser realizada com segurança, satisfazendo assim o resultado pretendido pelo cirurgião e paciente quanto à forma e às cicatrizes.
\end{abstract}

Descritores: Axila. Mamoplastia. Mama. Próteses e implantes. Cirurgia plástica.

\begin{abstract}
Introduction: The surgery for augmentation breasts developed in the last years, as well as the degree of requirement of our patients, in relation to the results related to the form of the breast and less apparent scars each time. Methods: Our experience initiated in November of 2002, with the possibility to utilize this technique in axillary access in a subfascial plan for increase of augmentation breasts of silicone implants. Results: This technique has been show to be a safety procedure with satisfying results for the surgeon and patient relating form and scars.
\end{abstract}

Keywords: Axilla. Mammaplasty. Breast. Prostheses and implants. Surgery, plastic.

\section{INTRODUÇÃO}

Existem várias técnicas que utilizam diferentes vias de acesso para aumento das mamas por meio de implantes mamários de silicone, com resultados satisfatórios.

Desde a descrição por Hoehler ${ }^{1}$, em 1973, e demonstrada por Tebbets ${ }^{2}$, a tentativa de se obter uma técnica de aumento de mamas via axilar demandava a introdução de implantes em plano retromuscular, devido a sua facilidade no acesso à mesma.

A mesma apresentava vários inconvenientes, desde a dificuldade em desinserção parcial do músculo peitoral, migração dos implantes no sentido cranial até assimetrias do sulco inframamário. Wallace et al. ${ }^{3}$, em seu trabalho de 1996, demonstraram aumento das queixas de dor em pacientes submetidas a aumento de mamas em plano retromuscular.

A dissecção romba com o dedo ou utilizando-se afastadores específicos, como uma válvula de Hegar proposta por Agris et al. ${ }^{4}$, não proporcionava descolamento seguro e hemostasia adequada.
Com o advento da cirurgia videoassistida, houve aumento da incidência de introdução de implantes em plano retroglandular.

Acreditamos como objetivo do nosso trabalho, que o acesso ao plano retroglandular pode ser realizado utilizandose de afastadores específicos, podendo assim atingir um resultado satisfatório, equilibrando implantes bem posicionados, plano adequado, simetria mamária e cicatrizes estéticas.

\section{MÉTODO}

Foram selecionadas 231 pacientes que apresentavam quadros de hipomastia, sem ptose associada, que desejavam aumento das mamas por meio de implantes de silicone por via axilar.

Utilizamos a técnica de mastoplastia de aumento via axilar ${ }^{1,2}$, em plano subfascial, entre novembro de 2002 e julho de 2006. A idade das pacientes variou de 17 a 45 anos e o volume de implantes mamários, de 220 a 500 cc.

1. Médico Cirurgião Plástico; Membro Titular da Sociedade Brasileira de Cirurgia Plástica, Clínica Privada do autor, São Paulo, SP, Brasil. 


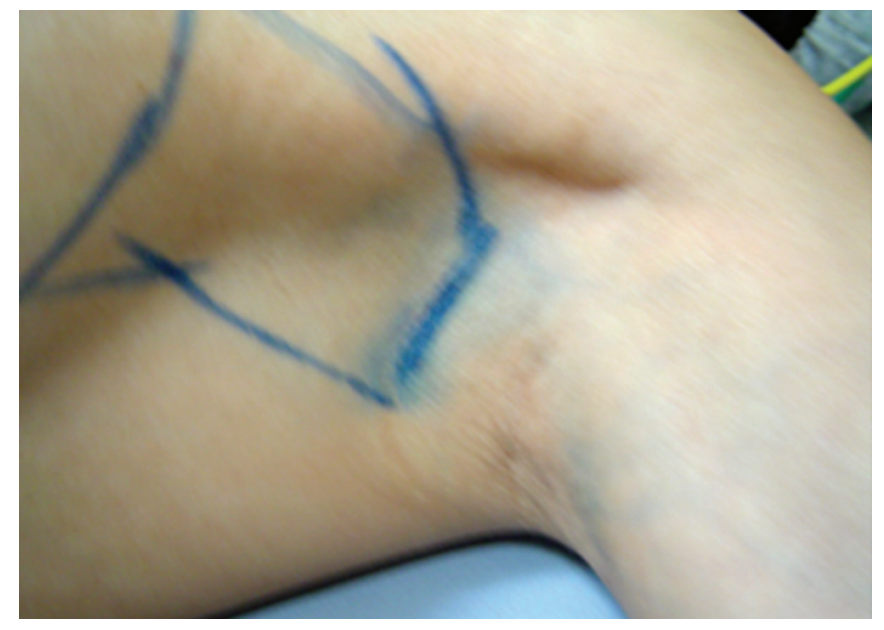

Figura 1 - Marcação cirúrgica no cavum axilar.

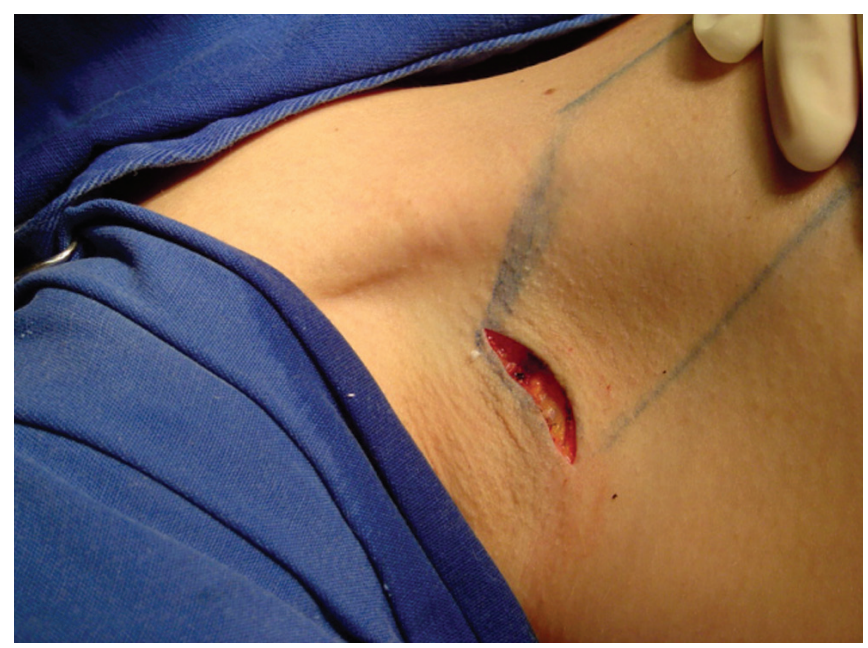

Figura 2 - Incisão de 3,5 cm em região axilar para acesso das mamas.

\section{Marcações}

Com a paciente em pé, marca-se a projeção do sulco inframamário bilateral e as dobras naturais nas axilas aonde será realizada a incisão em torno de 3 a $4 \mathrm{~cm}$, para acesso cirúrgico das mamas (Figura 1).

Com a paciente deitada, marca-se a delimitação das mamas e o túnel por onde será introduzido o implante, tendo como limite superior a borda súpero-lateral do músculo peitoral maior e, como limite inferior, o cavum axilar lateral.

\section{Técnica Cirúrgica}

Com a paciente em decúbito dorsal horizontal, sob anestesia peridural e sedação, realiza-se antissepsia com clorexidina $^{\circledR}$ degermante e, em seguida, clorexidina ${ }^{\circledR}$ alcoólica, coloca-se os campos cirúrgicos e inicia-se a cirurgia.

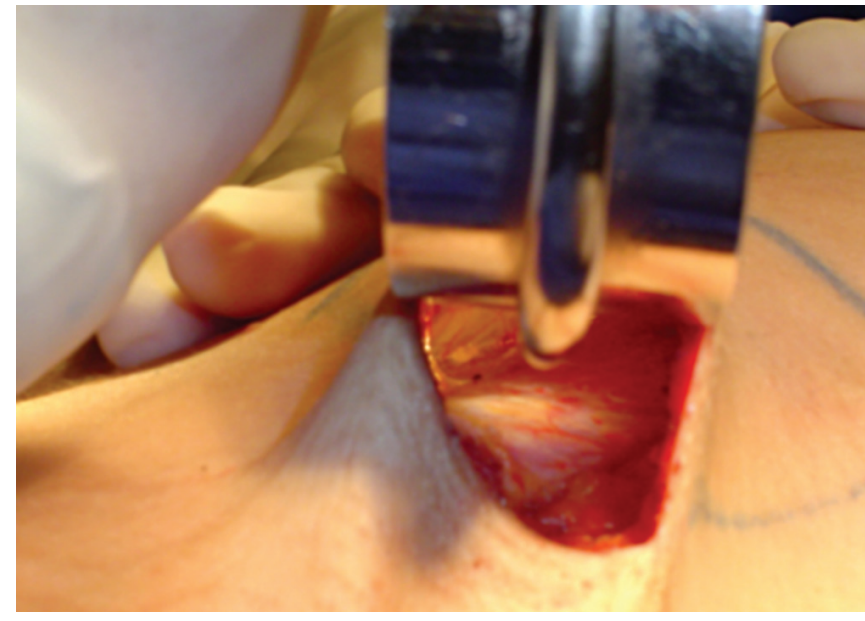

Figura 3 - Visualização do músculo peitoral maior para descolamento subfascial.

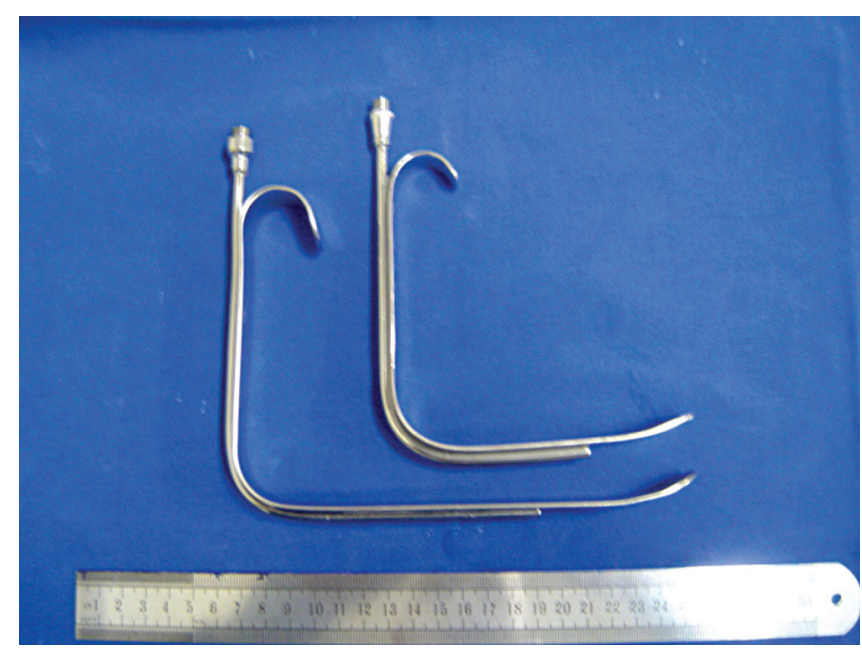

Figura 4-Diferença entre o afastador padrão e o afastador longo de fibra óptica.

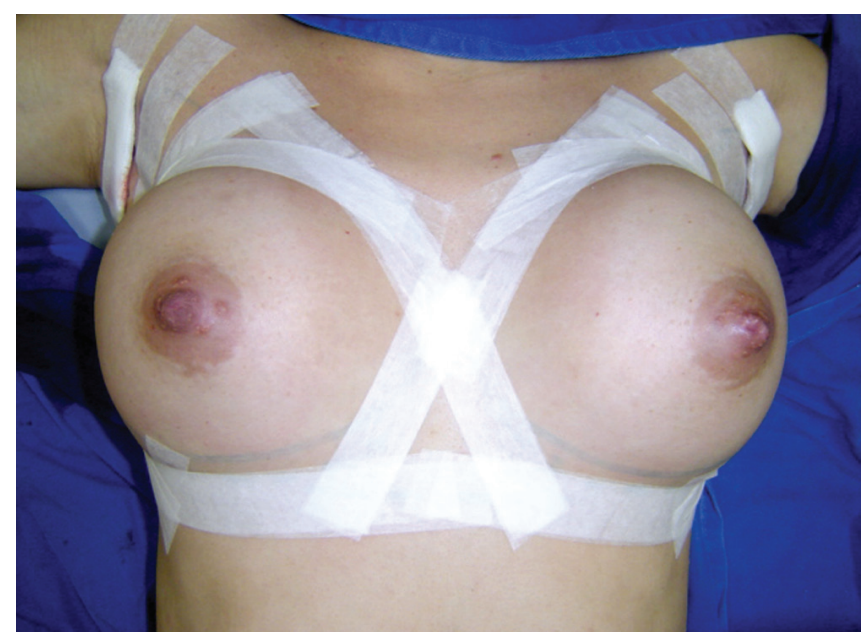

Figura 5-Curativo com micropore ${ }^{\circledR}$ para contenção das mamas. 
O primeiro passo é a incisão em região axilar em torno de 3,5 cm de comprimento (Figura 2).

Após divulsão delicada, parte-se em sentido cranial numa angulação em torno de 45 graus até identificação do músculo peitoral superior (Figura 3). Sendo assim, inicia-se o descolamento caudal, acima do plano muscular e abaixo da fáscia, em direção ao sulco inframamário por visualização direta por meio de afastadores com fibra óptica. Vale salientar a utilização de afastadores mais longos iluminados com fibra óptica, a partir do descolamento da porção mediana da mama, sendo este também feito sob visão direta (Figura 4).

Após confecção da loja para introdução do implante e hemostasia rigorosa, realizada sob visão direta, parte-se para introdução do implante mamário. Utilizando-se dois afastadores de Farabeuf, introduz-se o implante mamário.

$\mathrm{O}$ mesmo procedimento é realizado na mama contralateral.

Ao posicionarmos adequadamente o implante no local desejado, parte-se para fechamento por planos, realizados

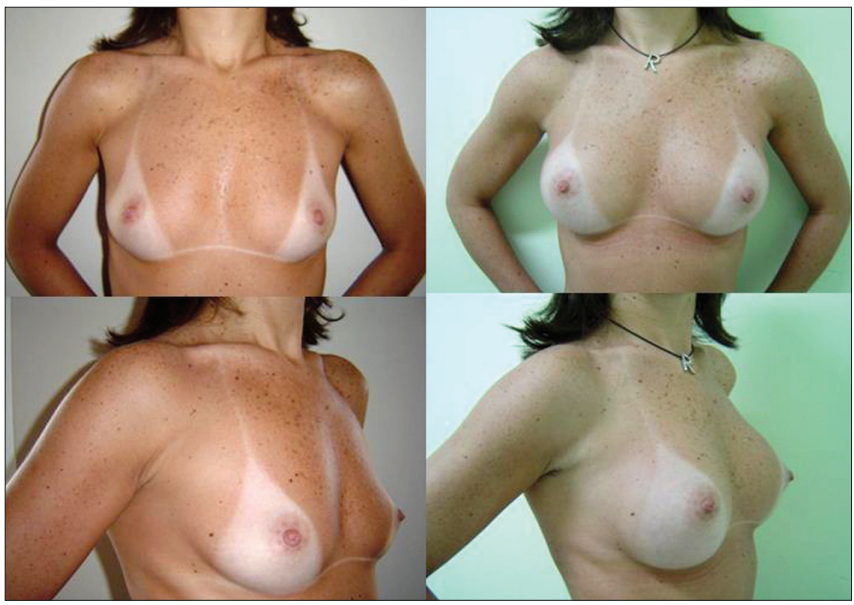

Figura 6-Resultado cirúrgico: pré e pós-operatório de 6 meses, $260 \mathrm{cc}$.

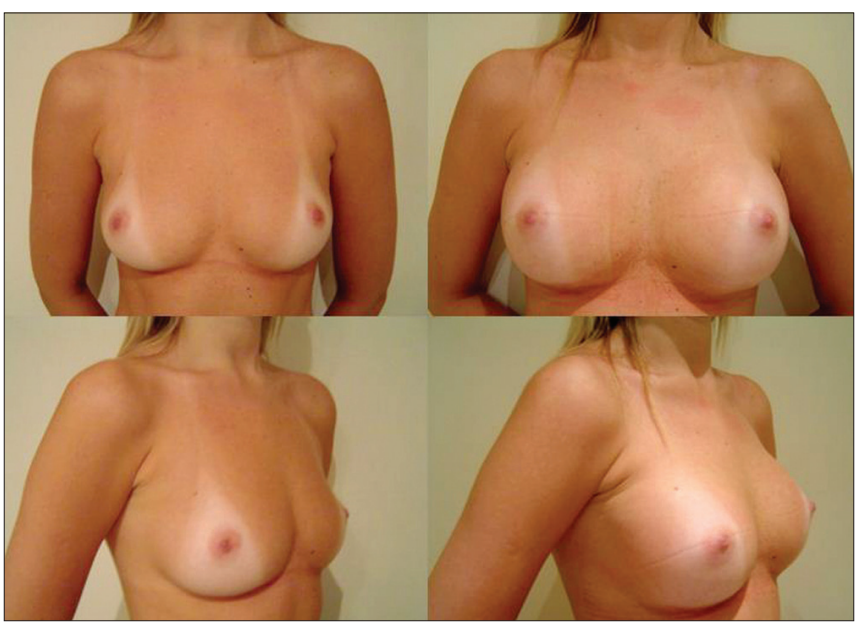

Figura 7-Resultado cirúrgico: pré e pós-operatório de 6 meses, 3000 cc.

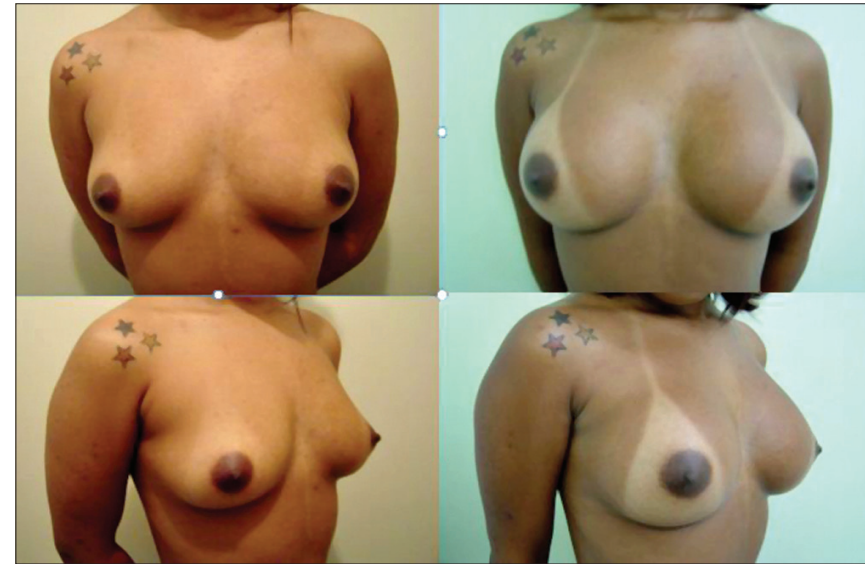

Figura 8-Resultado cirúrgico: pré e pós-operatório de 6 meses, 260 cc à direita e 220 cc à esquerda.

por fechamento subdérmico com monocryl $1^{\circledR} 4-0$ e sutura intradérmica com mononylon 4-0.

Após realização do procedimento nos dois lados, parte-se para o curativo confeccionado com micropore ${ }^{\circledR}$ ao redor das mamas (Figura 5), seguido de compressas e faixa elástica no pólo superior das mamas, com tração caudal e sutiã cirúrgico.

\section{RESULTADOS}

A partir de 2002, operamos 231 pacientes portadoras de hipomastia bilateral, pela técnica de mastoplastia de aumento via axilar, em plano subfascial (Figuras 6 a 8). Em 208 (90\%)

\begin{tabular}{c|c|c|c|c}
\hline \multicolumn{5}{c}{ Tabela $1-\begin{array}{c}\text { Volume dos implantes utilizados, em valores } \\
\text { unitários, por mama operada. }\end{array}$} \\
\hline $\begin{array}{c}\text { Volume } \\
\text { das } \\
\text { Próteses }\end{array}$ & $\begin{array}{c}\text { Mama } \\
\text { Direita }\end{array}$ & $\begin{array}{c}\text { Mama } \\
\text { Esquerda }\end{array}$ & Total & $\%$ \\
\hline $200 \mathrm{cc}$ & & $1 / 462$ & $1 / 462$ & $0,2 \%$ \\
\hline $220 \mathrm{cc}$ & $5 / 462$ & $8 / 462$ & $13 / 462$ & $2,81 \%$ \\
\hline $230 \mathrm{cc}$ & $4 / 462$ & $4 / 462$ & $8 / 462$ & $1,73 \%$ \\
\hline $240 \mathrm{cc}$ & $20 / 462$ & $17 / 462$ & $37 / 462$ & $8 \%$ \\
\hline $260 \mathrm{cc}$ & $50 / 462$ & $47 / 462$ & $97 / 462$ & $21 \%$ \\
\hline $280 \mathrm{cc}$ & $60 / 462$ & $59 / 462$ & $119 / 462$ & $25,75 \%$ \\
\hline $300 \mathrm{cc}$ & $43 / 462$ & $46 / 462$ & $89 / 462$ & $19,26 \%$ \\
\hline $325 \mathrm{cc}$ & $9 / 462$ & $9 / 462$ & $18 / 462$ & $3,9 \%$ \\
\hline $350 \mathrm{cc}$ & $26 / 462$ & $26 / 462$ & $52 / 462$ & $11,25 \%$ \\
\hline $375 \mathrm{cc}$ & $5 / 462$ & $5 / 462$ & $10 / 462$ & $2,16 \%$ \\
\hline $400 \mathrm{cc}$ & $5 / 462$ & $5 / 462$ & $10 / 462$ & $2,16 \%$ \\
\hline $450 \mathrm{cc}$ & $2 / 462$ & $2 / 462$ & $4 / 462$ & $0,8 \%$ \\
\hline $500 \mathrm{cc}$ & $2 / 462$ & $2 / 462$ & $4 / 462$ & $0,8 \%$ \\
\hline & & & &
\end{tabular}




\begin{tabular}{l|c|c}
\hline \multicolumn{3}{c}{ Tabela 2 - Cirurgias associadas. } \\
\hline $\begin{array}{l}\text { Cirurgias } \\
\text { Associadas }\end{array}$ & $\begin{array}{c}\text { Número de } \\
\text { Pacientes }\end{array}$ & Porcentagem \\
\hline Lipoescultura & $19 / 231$ & $8,2 \%$ \\
\hline Rinoplastia & $6 / 231$ & $2,6 \%$ \\
\hline Dermo abdominal & $4 / 231$ & $1,73 \%$ \\
\hline Otoplastia & $2 / 231$ & $0,86 \%$ \\
\hline Mini abdome & $2 / 231$ & $0,86 \%$ \\
\hline Blefaroplastia & $1 / 231$ & $0,43 \%$ \\
\hline Total & $34 / 231$ & $14,71 \%$ \\
\hline
\end{tabular}

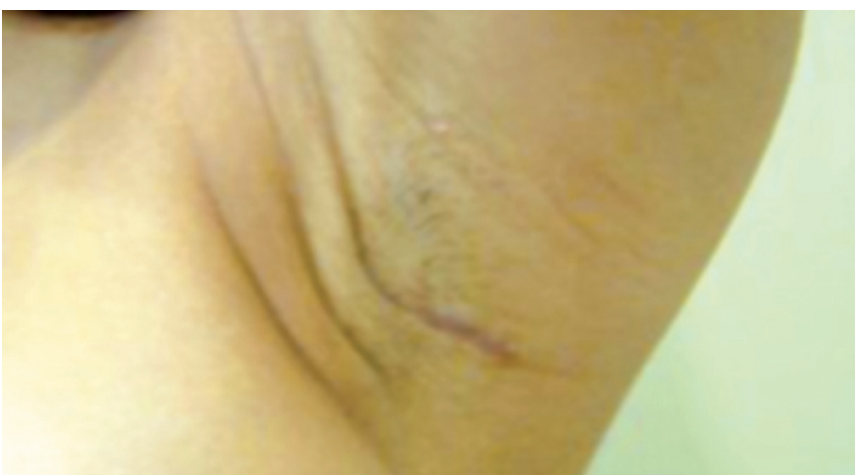

Figura 9 - Retração cirúrgica: retração axilar resolvida com massagem local.

Tabela 3 - Complicações pós-operatórias.

\begin{tabular}{l|c|c}
\hline Complicações & $\begin{array}{c}\text { Número de } \\
\text { Pacientes }\end{array}$ & Porcentagem \\
\hline Seroma unilateral & $2 / 231$ & $0,86 \%$ \\
\hline Contratura capsular unilateral & $4 / 231$ & $1,73 \%$ \\
\hline Hematoma unilateral & $2 / 231$ & $0,86 \%$ \\
\hline Aderência axilar unilateral & $2 / 231$ & $0,86 \%$ \\
\hline Estrias bilaterais & $2 / 231$ & $0,86 \%$ \\
\hline Total & $12 / 231$ & $5,2 \%$ \\
\hline
\end{tabular}

pacientes, introduzimos implantes do mesmo volume e, em $23(10 \%)$, utilizamos implantes de volumes diferentes. O volume dos implantes variou de $200 \mathrm{ml}$ a $500 \mathrm{ml}$, sendo que $65,91 \%$ ficaram na faixa entre $260 \mathrm{cc}$ a $300 \mathrm{cc}$, tendo sua distribuição descrita na Tabela 1.

Ocorreram 4 casos de contratura capsular unilateral, 2 casos de hematoma unilateral que foram reoperados no pós-operatório imediato, 2 casos de estrias periareolares, 2 casos de seroma unilateral e 2 casos de aderência na cicatriz axilar que foram resolvidos com massagem local (Tabela 2, Figura 9). Perda de sensibilidade parcial no complexo aréolo-mamilar, quando presente, foi transitória. Não houve casos de migração dos implantes, infecção,

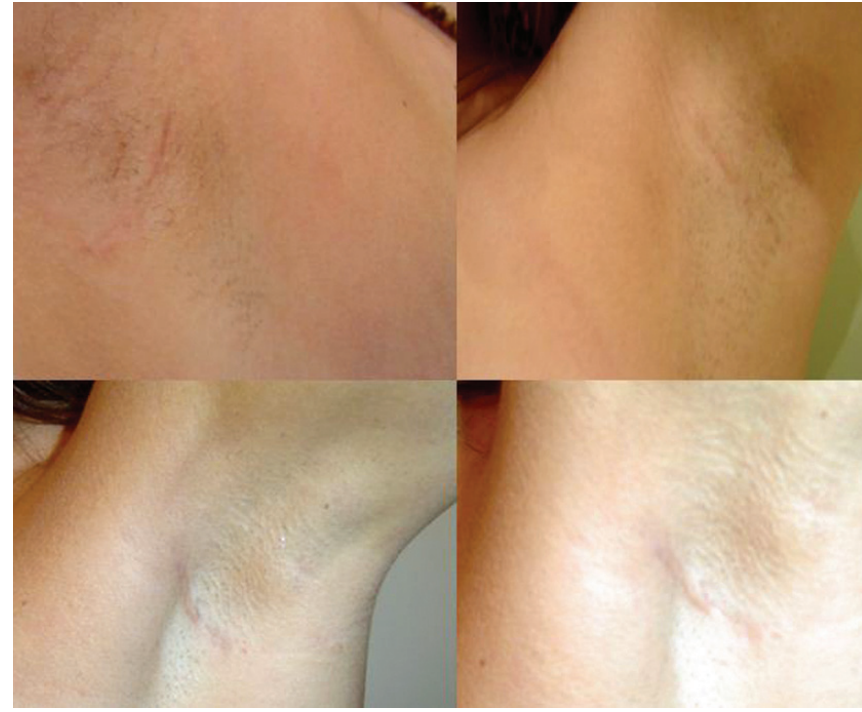

Figura 10 - Cicatrizes cirúrgicas em cavum axilar.

deiscência ou necroses. As cirurgias associadas estão discriminadas na Tabela 3.

Das 231 pacientes, 2 ficaram grávidas, tiveram seus filhos e conseguiram amamentar sem nenhum problema. Uma paciente evoluiu com ptose após 3 anos de cirurgia e necessitou de pexia mamária.

\section{DISCUSSÃO}

A técnica para aumento de mamas por via axilar, em plano subfascial, tem permitido um acesso adequado, com boa visualização na confecção da loja em que será introduzida o implante mamário, com adequada hemostasia, sendo muito segura, mesmo utilizando-se implantes com volumes maiores.

A vantagem desta técnica em relação à mesma via de acesso em plano retromuscular consiste numa menor chance de deslocamento do implante, porém tendo a desvantagem em pacientes que não possuem uma cobertura cutânea adequada para o implante mamário.

Consideramos contraindicação para esta via de acesso, pacientes que apresentam mamas tuberosas ou que necessitem de pexia mamária para correção de ptose.

As complicações encontradas são semelhantes a outras vias de acesso, ou seja, inerentes à especificidade desta via de acesso.

A aceitação e o desejo das pacientes em relação à via de acesso axilar vêm crescendo ano a ano, devido ao posicionamento das cicatrizes (Figura 10), qualidade das mesmas e aspecto de "mamas sem cicatrizes" desejado por nós e pelas pacientes.

A pesquisa do linfonodo sentinela após manipulação da axila em cirurgias para aumento das mamas não parece ser 
um fator limitante na utilização desta técnica ${ }^{7,8}$, podendo ser realizada mesmo após o procedimento cirúrgico, principalmente em plano subfascial. Alguns estudos sugerem uma avaliação com um tempo de pós-operatório mais longo, para termos uma avaliação mais precisa ${ }^{7}$.

A palpação de tumores parece ser facilitada em pacientes previamente submetidas à cirurgia de implantes mamários em comparação às não operadas 9 .

Sendo assim, o diagnóstico e o estadiamento adequados em pacientes com implantes mamários não dependem exclusivamente da pesquisa do linfonodo sentinela, mas sim da somatória de exames que incluem: mamografia, ultrassonografia das mamas, ressonância nuclear magnética das mamas e biopsia de agulha fina ${ }^{10}$.

\section{CONCLUSÃO}

A técnica de mastoplastia de aumento via axilar, subfascial, tem se mostrado segura, com ótimos resultados e larga aplicação. A utilização de afastadores iluminados com fibra óptica mais longos, de fácil manuseio, facilita a visualização e, consequentemente, a confecção da loja cirúrgica e hemostasia adequada.

Desta forma, ficamos convencidos e seguros na indicação desta técnica para aumento das mamas.

\section{REFERÊNCIAS}

1. Hoehler H. Further progress in the axillary approach in augmentation mammaplasty: prevention of encapsulation. Aesthetic Plast Surg. 1977;1:107.

2. Tebbetts JB. Transaxillary subpectoral augmentation mammaplasty: A critical appraisal with long-term follow-up and refinements. In: Aesthetic Plastic Surgery. Los Angeles, 1983.

3. Wallace MS, Wallace AM, Lee J, Dobke MK. Pain after breast surgery: a survey of 282 women. Pain.1996;66(2-3):195-205.

4. Agris J, Dingman RO, Wilensky RJ. A dissector for the transaxillary approach in augmentation mammaplasty. Plast Reconstr Surg. 1976;57(1):10-3.

5. Hudson DA. Submuscle saline breast augmentation: are we making sense in the new millenium? Aesthetic Plast Surg. 2002;26(4):287-90.

6. Pereira LH, Sterodimas A. Transaxillary breast augmentation: a prospective comparison of subglandular, subfascial, and submuscular implant insertion. Aesthetic Plast Surg. 2009;33(5):752-9.

7. Munhoz AM, Aldrighi C, Buschpiegel C, Ono C, Montag E, Fells K, et al. The feasibility of sentinel lymph node detection in patients with previous transaxillary implant breast augmentation: preliminary results. Aesthetic Plast Surg. 2005;29(3):163-8.

8. Port ER, Fey J, Gemignani ML, Heerdt AS, Montgomery LL, Petrek JA, et al. Reoperative sentinel lymph node biopsy: a new option for patients with primary or locally recurrent breast carcinoma. J Am Coll Surg. 2002;195(2):167-72.

9. Skinner KA, Silberman H, Dougherty W, Gamagami P, Waisman J, Sposto R, et al. Breast cancer after augmentation mammoplasty. Ann Surg Oncol. 2001;8(2):138-44.

10. Kulber DA, Mackenzie D, Steiner JH, Glassman H, Hopp D, Hiatt JR, et al. Monitoring the axilla in patients with silicone gel implants. Ann Plast Surg. 1995;35(6):580-4. 